\title{
Análise de desconforto térmico local em ambientes cirúrgicos com o uso de manequim, medição de variáveis ambientais e avaliação subjetiva
}

\author{
Analisys of local thermal discomfort in surgical \\ environments with the use of mannequins, measurement \\ of environmental variables and subjective evaluation
}

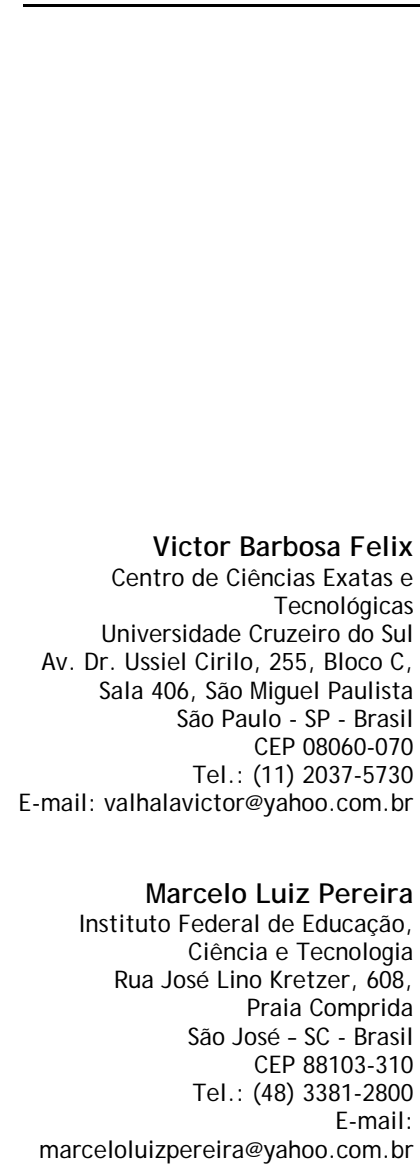

\section{Victor Barbosa Felix \\ Marcelo Luiz Pereira \\ Danilo de Moura \\ Arlindo Tribess}

\section{Resumo}

$\mathbf{N}$

a avaliação de conforto térmico em ambientes interiores não basta analisar as condições de conforto para o corpo como um todo, pois há a necessidade de se analisar também as condições de desconforto térmico local. Em ambientes complexos, tais como os ambientes cirúrgicos, onde os membros da equipe cirúrgica ocupam diferentes posições no ambiente e desempenham atividades distintas, a análise de condições de desconforto térmico local torna-se ainda mais premente. No presente trabalho foram analisadas condições de desconforto térmico local devido a assimetrias da temperatura radiante, diferença vertical de temperatura do ar e risco de correntes de ar utilizando manequim, medição de variáveis ambientais e avaliação subjetiva. Resultados da avaliação subjetiva mostraram níveis de insatisfação de até 35 \% dos anestesistas e enfermeiros com correntes de ar e de até 85\% dos cirurgiões com o calor do foco cirúrgico. Resultados similares foram obtidos a partir da medição de variáveis ambientais e com o uso de manequim. Estes resultados ressaltam ainda mais a grande dificuldade de se prover condições de conforto térmico neste tipo de ambiente. Entretanto, a utilização de diferentes ferramentas de análise pode auxiliar na busca de se prover condições de conforto térmico as melhores possíveis para todos os membros da equipe cirúrgica.

Palavras-chave: Desconforto térmico local. Manequins. Temperaturas equivalentes. Avaliação subjetiva. Ambientes cirúrgicos.

\section{Abstract}

In the evaluation of thermal comfort in indoor environments it is not enough to perform the analysis of the comfort conditions for the body as a whole, since it is also necessary to examine the conditions of local thermal discomfort. In complex environments, such as surgical environments, where team members occupy

Danilo de Moura Centro de Ciências Exatas e Tecnológicas Universidade Cruzeiro do Sul E-mail: dm.termica@gmail.com

Arlindo Tribess

Departamento de Engenharia

Mecânica, Escola Politécnica

Universidade de São Paulo Av. Prof. Mello Moraes, 2231, Campus Universitário, Butantã São Paulo - SP - Brasil
CEP 05508-900

Tel.: (11) 3091-5355 E-mail: atribess@usp.br

different positions in the surgical environment and perform distinct activities, an analysis of the conditions of local thermal discomfort becomes even more necessary. This study analysed local thermal discomfort conditions due to radiant temperature asymmetry, vertical differences in air temperature and the risk of draughts through the use of mannequins, measurements of environmental variables and subjective evaluation. Results of the subjective evaluation showed dissatisfaction levels of up to 35\% of anaesthesiologists and nurses regarding draughts, and up to 85\% of surgeons regarding the heat of the surgical focus. Similar results were obtained from the measurement of environmental variables and the use of mannequins. These results confirm the great difficulty of providing thermal comfort in such an environment. However, the use of different analysis tools can assist in finding the best possible thermal comfort conditions for all members of the surgical team.

Recebido em 06/10/11 Keywords: Local thermal discomfort. Mannequins. Equivalent temperatures. Subjective Aceito em 06/03/12 evaluation. Surgical environments. 


\section{Introdução}

Hospitais e demais instalações médicas constituem-se em ambientes complexos que requerem ventilação adequada para o controle de contaminação aérea e para o conforto de pacientes, de pessoal e de visitantes.

Essa maior complexidade dos ambientes hospitalares requer que os sistemas de tratamento de ar e de ventilação possuam funções mais abrangentes que aquelas de outros tipos de edificações. Nesse caso, envolvem a proteção de funcionários e pacientes suscetíveis a agentes patogênicos transportados pelo ar, a minimização dos riscos de transmissão de agentes patogênicos transportados pelo ar por pacientes infectados, a remoção e controle de odores, e a manutenção da temperatura e da umidade do ar no recinto em níveis confortáveis para os funcionários, pacientes e visitantes (AMERICAN..., 1996).

Em ambientes cirúrgicos, a necessidade ainda mais premente de diminuir os riscos de infecções dos pacientes e dos profissionais de saúde (BEGGS, 2003; HOWORTH, 1993; WHYTE et al., 1992; WHYTE; HODGSON; TINKLER, 1982), na maioria das vezes coloca o conforto térmico em segundo plano, embora condições de conforto do cirurgião e da equipe médica necessitem ser as melhores possíveis para que trabalhem em condições favoráveis para o sucesso do procedimento cirúrgico. Adicionalmente, é necessário atenção para as condições do paciente, para que condições desfavoráveis de conforto térmico não comprometam ainda mais seu estado de saúde (FELIX, 2008).

Outro aspecto importante a considerar-se é a forma como o ar movimenta-se no interior da sala cirúrgica. A movimentação do ar terá um padrão característico do sistema de insuflamento utilizado com perfis de temperatura e velocidade completamente diferentes daqueles verificados em outros ambientes com outros tipos de sistemas de ventilação (PEREIRA; TRIBESS, 2005), que influenciam diretamente no risco de contaminação aérea e no conforto térmico da equipe cirúrgica e do paciente.

Prover condições de conforto térmico para os membros da equipe cirúrgica não é tarefa fácil. Em trabalho anterior dos autores do presente artigo (FELIX et al., 2010), foram apresentados resultados de estudo comparativo de condições de conforto térmico em salas cirúrgicas a partir dos resultados de medição de variáveis ambientais obtidos por Felix (2008), Mora, English e Athienitis (2001) e Wyon, Lidwell e Willians (1968). Os resultados daquele estudo mostraram valores de temperaturas equivalentes, $\mathrm{T}_{\mathrm{eq}}$, de conforto térmico praticamente iguais nos três trabalhos, em torno de $22{ }^{\circ} \mathrm{C}$ para todos os membros da equipe cirúrgica, com $\mathrm{T}_{\text {eq }}$ dado pela Equação 1 (MADSEN; OLESEN; KRISTENSEN, 1984):

$T_{e q}=0,55 \cdot T_{a r}+0,45 \cdot \overline{T_{r}}+\frac{0,24-0,75 \cdot \sqrt{V_{a r}}}{1+I_{R}} \cdot\left(36,5-T_{a r}\right)$ Eq. 1

Onde:

$\mathrm{T}_{\text {eq }}=$ temperatura equivalente $\left[{ }^{\circ} \mathrm{C}\right]$;

$\mathrm{V}_{\mathrm{ar}}=$ velocidade média do ar $[\mathrm{m} / \mathrm{s}]$;

$\bar{T}_{r}=$ temperatura radiante média $\left[{ }^{\circ} \mathrm{C}\right]$;

$\mathrm{T}_{\mathrm{ar}}=$ temperatura do $\mathrm{ar}\left[{ }^{\circ} \mathrm{C}\right]$; e

$\mathrm{I}_{\mathrm{R}}=$ índice de isolamento total da roupa [clo].

Esse resultado é particularmente útil, pois permite juntamente com a utilização da Equação 1, avaliar diferentes condições ambientais e pessoais em propiciar condições de conforto térmico aos diferentes membros da equipe cirúrgica.

Paralelamente, resultados apresentados em Felix et al. (2010) mostraram que a aplicação do método de Fanger (1972) também é adequado na avaliação de condições de conforto térmico em salas cirúrgicas, embora seja necessário cuidado especial em sua utilização e na análise dos resultados.

É importante ressaltar que as temperaturas equivalentes da Equação 1 e os resultados do método de Fanger (1972) referem-se à análise de condições de conforto térmico para o corpo como um todo. Mas, mesmo que sejam satisfeitas as condições de conforto ou de desconforto térmico dentro dos limites aceitáveis previstos nas normas ASHRAE 55 (AMERICAN..., 2004), ISO 7730 (INTERNATIONAL..., 2005) e NBR 16401-2 (ABNT, 2008), insatisfações térmicas locais podem ocorrer ocasionando sensação de "frio" ou “calor” em uma parte particular do corpo.

Neste caso, a ASHRAE 55 (INTERNATIONAL..., 2004), a ISO 7730 (INTERNATIONAL..., 2005) e a NBR 16401-2 (ABNT, 2008) recomendam limites adicionais para a aceitação do ambiente baseados em um critério de $5 \%$ a $15 \%$ de insatisfação. Esses percentuais não são aditivos, pois pessoas que sentem conforto ou desconforto podem sentir ou não desconforto localizado e de modos diferentes. Sendo assim, a norma admite que um ambiente estará confortável, sob os dois pontos de vista, se satisfizer a pelo menos $80 \%$ dos ocupantes. 
Em complementação ao artigo de Felix et al. (2010) de análise de condições de conforto térmico em ambientes cirúrgicos, o objetivo do presente trabalho é avaliar as condições de desconforto térmico local nesses tipos de ambientes com o uso de manequim, medição de variáveis ambientais e avaliação subjetiva.

\section{Desconforto térmico local}

O desconforto térmico local ocorre devido a fatores que alteram a uniformidade no ambiente. Tais fatores podem ser devido a correntes de ar, diferença vertical de temperatura do ar, janelas ou superfícies frias ou quentes, ou variações deles.

Quando ocorre um resfriamento localizado e indesejado no corpo, devido ao movimento do ar, há uma sensação de corrente de ar, que depende da velocidade do ar, da temperatura do ar, da intensidade de turbulência, da atividade e da vestimenta das pessoas.

A sensibilidade a correntes de ar é maior em partes do corpo descobertas, especialmente na região da cabeça, pescoço e ombros e na região dos pés, tornozelos e pernas, e em atividade leve ou sedentária. Assim, a ocorrência de correntes de ar deve ser analisada principalmente na altura da cabeça e tronco das pessoas $(1,10 \mathrm{~m}$ do piso para pessoas sentadas e $1,70 \mathrm{~m}$ para pessoas em pé). Se a pessoa estiver com as pernas descobertas, deve ser feita a verificação também no nível a 0,10 m do piso.

Segundo Fanger et al. (1988), para atividade sedentária ou leve, o percentual de insatisfação (DR) com as correntes de ar é dada pela Equação 2 .

$D R=\left\lfloor\left(34-T_{a r}\right) \cdot\left(V_{a r}-0,05\right)^{0,62}\right\rfloor\left(0,37 \cdot V_{a r} \cdot T_{u}+3,14\right)$

Eq. 2

Onde:

$\mathrm{T}_{\mathrm{ar}}=$ temperatura do ar local $\left[{ }^{\circ} \mathrm{C}\right]$;

$\mathrm{V}_{\mathrm{ar}}=$ velocidade média do ar local [m/s]; e
$\mathrm{T}_{\mathrm{u}}=$ intensidade de turbulência [\%].

Na Equação 2 a intensidade de turbulência $\left(T_{u}\right)$ é a relação entre o desvio padrão $\left(\mathrm{DP}_{\mathrm{V}}\right)$ e a média da velocidade do ar $\left(\mathrm{V}_{\mathrm{a}}\right)$, expressa em porcentagem. Essa relação pode ser calculada pela Equação 3, com base nos valores de velocidade do ar, medidos com intervalos de 0,2 s durante, pelo menos, 3 min, e o desvio padrão $\left(\mathrm{DP}_{\mathrm{V}}\right)$, referente à respectiva coleção de dados.

$T_{u}=\frac{D P_{V}}{V_{a r}} \cdot 100$

Eq. 3

\section{Diferença vertical de temperatura do ar}

Geralmente, em ambientes interiores a temperatura do ar aumenta do piso para o teto. Se esse aumento é muito grande, pode ocorrer desconforto local expresso por calor na cabeça e frio nos pés, embora o corpo como um todo possa estar em situação de conforto. As normas técnicas preveem limite máximo de $3{ }^{\circ} \mathrm{C}$ de diferença de temperatura entre os pés e a cabeça.

\section{Assimetria da temperatura radiante}

A radiação térmica em torno do ocupante pode não ser uniforme, devido tanto a superfícies frias ou quentes quanto à radiação solar direta. Essa assimetria pode causar desconforto local e reduzir a aceitabilidade térmica do ambiente. Em geral, as pessoas são mais sensíveis à radiação assimétrica causada por teto quente do que por superfícies verticais frias ou quentes.

A medida da assimetria da temperatura radiante plana é dada pela diferença entre as temperaturas radiantes planas de dois lados opostos de um elemento plano pequeno. Deve ser medida na altura 0,60 m para pessoas sentadas e a 1,10 $\mathrm{m}$ do piso, para pessoas em pé. Para indivíduos desenvolvendo atividade sedentária ou leve, os limites para a assimetria da temperatura radiante são apresentados na Figura 1. 


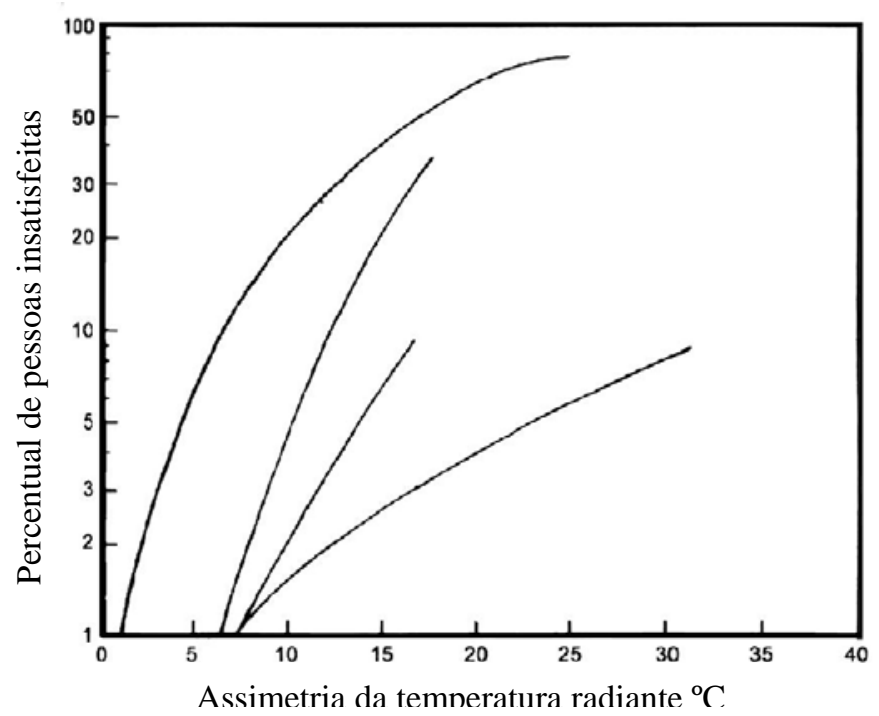

Figura 1 - Percentual de pessoas insatisfeitas devido à assimetria da temperatura radiante

Fonte: ASHRAE (AMERICAN..., 2009).

\section{Temperatura do piso}

Ocupantes de ambientes interiores podem sentir desconforto nos pés, mesmo calçados, devido ao contato direto com o piso, se este estiver frio ou quente. O desconforto com a temperatura do piso é maior em climas frios, com temperaturas mais baixas do piso (ASHRAE, 2009).

A forma convencional de análise de condições de desconforto térmico local consiste na medição de variáveis ambientais (temperaturas e velocidades do ar, temperaturas de superfícies e de assimetrias da temperatura radiante), com cuidados $\mathrm{e}$ procedimento preconizados na norma ISO 7726 (INTERNATIONAL..., 1998). Feitas as medições, faz-se a análise dos resultados utilizando equações e figuras do subitem anterior ou valores recomendados nas normas ASHRAE 55 (AMERICAN..., 2004), ISO 7730 (INTERNATIONAL..., 2005) e NBR 16401-2 (ABNT, 2008). Além disso, outra forma usualmente utilizada é aquela da análise do percentual de insatisfação a partir de resultados da aplicação de questionários.

Uma terceira forma, não usual em edificações, mas de uso corrente na indústria automobilística, é a de avaliação do efeito combinado dos diversos fatores ambientais no conforto térmico com o uso de manequins. Neste caso, tem-se a avaliação simultânea do conforto térmico para o corpo como um todo, similar ao método de Fanger, e do desconforto local, pois se está avaliando as condições de conforto térmico em cada segmento do corpo.

\section{Análise do desconforto térmico local com o uso de manequins}

Os manequins utilizados para a avaliação de conforto térmico podem ser manequins térmicos, manequins com sensores aquecidos e até mesmo manequins que não passam de pedaços de madeira reproduzindo o corpo humano, dotados de sensores, os sticks (ISO 14505-2 (INTERNATIONAL..., 2004).

Os manequins térmicos foram originalmente desenvolvidos para medir-se a isolação de roupas, mas são utilizados também para medirem-se as condições de conforto em ambientes com condições ambientais não uniformes, como, por exemplo, em veículos automotivos e em ambientes de edificações com assimetrias de temperatura e velocidade significativas.

Por serem equipados com aquecimento, os manequins térmicos permitem que a mesma perda de calor e temperaturas da pele de um ser humano possam ser simuladas. Normalmente, cada segmento do corpo possui sistema de controle de aquecimento próprio, e a troca de calor do manequim é realizada de maneira similar à do corpo humano, por condução, convecção e radiação térmica.

Existem basicamente três tipos de sistemas de aquecimento para os manequins, estando a diferença na localização dos elementos para o aquecimento:
(a) na superfície externa do manequim;
(b) na superfície interna; e
(c) dentro do corpo do manequim. 
Os manequins podem ser utilizados tanto para a avaliação das condições de conforto quanto para a avaliação do efeito da temperatura superficial na hipotermia de pacientes (CASSEY et al., 2004; FELIX et al., 2005).

Nilsson (2004) apresenta um estudo detalhado da utilização de manequins térmicos. Os manequins atualmente utilizados são divididos em 16 zonas (Figura 2), ou mais, até um limite de 32 zonas, independentemente controladas. Para reduzir custos e peso são utilizados materiais como alumínio e plástico.

A maioria dos manequins térmicos atuais só permite determinar a perda de calor sensível. Em condições ambientais onde o ser humano começar a suar para perder calor por evaporação haverá uma subestimação do calor total perdido. Entretanto, para pesquisas de conforto térmico realizadas próximo da zona de neutralidade térmica em atividades leves, onde a perda de calor por evaporação de suor é uma parte pequena da perda de calor total, não haverá um desvio muito grande se for utilizado um manequim térmico que não seja capaz de reproduzir a sudorese humana.

No caso de manequins com sensores aquecidos, o manequim não é aquecido como no manequim térmico. Em vez de fazer-se a avaliação das trocas de calor em diversos segmentos do corpo aquecido, são colocados sensores aquecidos nesses segmentos do corpo (normalmente 16 ou 32 segmentos).

Embora a utilização de manequins aquecidos seja mais adequada, por reproduzir mais fielmente as trocas de calor do corpo humano, o uso de manequins com sensores aquecidos, também previsto na norma ISO 14505-2 (INTERNATIONAL..., 2004), tem a vantagem de apresentar um custo significativamente menor que os manequins aquecidos e também de conseguir captar o efeito combinado dos diversos fatores ambientais no desconforto térmico local. Um exemplo desse manequim, utilizado no presente trabalho, é apresentado na Figura 3.
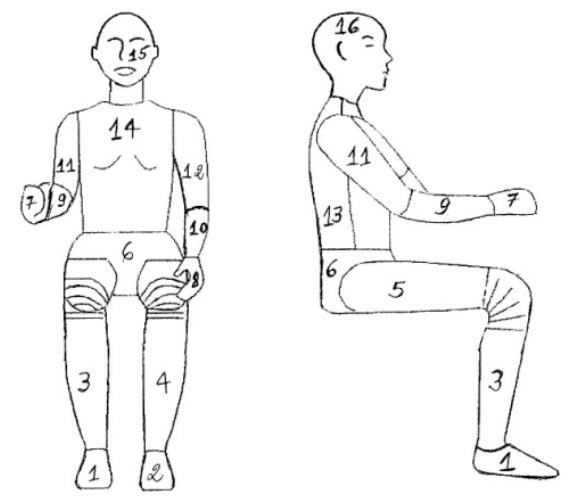

Figura 2 - Manequim com 16 segmentos

Fonte: ISO 14505 (ABNT, 2004).

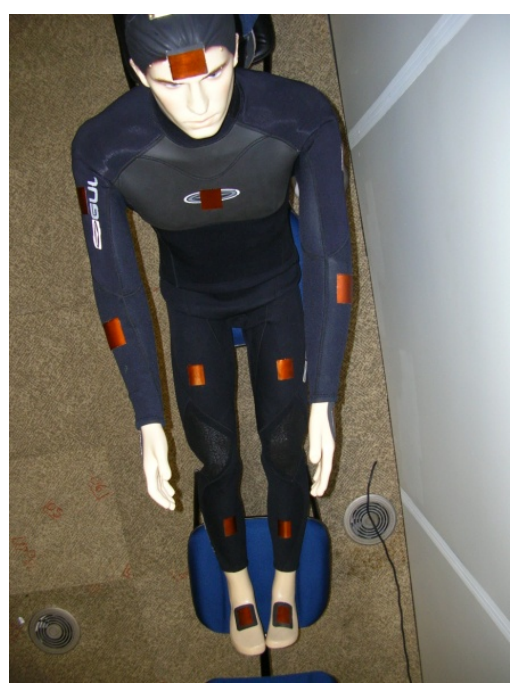

Figura 3 - Manequim com sensores aquecidos 


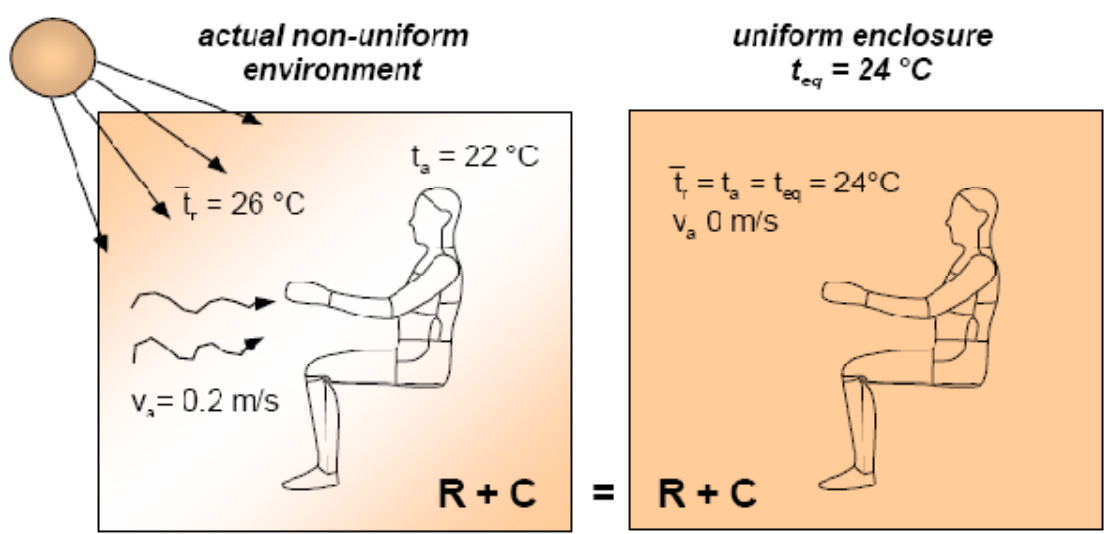

Figura 4 - llustração da definição de temperatura equivalente aplicada aos diversos segmentos do corpo Fonte: adaptada de Nilsson (2004).

Para ambientes onde diferentes partes do corpo experimentam diferentes condições térmicas, o conceito mais amplamente utilizado para avaliação de condições de conforto térmico (GAMEIRO DA SILVA, 2002; NILSSON, 2004) também é o de temperatura equivalente $\left(\mathrm{T}_{\mathrm{eq}}\right)$, definida como sendo a temperatura de um invólucro imaginário, com a temperatura radiante média igual à temperatura do ar e ar parado, no qual a pessoa troca a mesma quantidade de calor por radiação e convecção que nas condições reais (Figura 4).

Para a determinação de temperaturas equivalentes em cada segmento do corpo não se utiliza a Equação 1 (corpo como um todo), mas procedimento constante da norma ISO 14505-2 (INTERNATIONAL..., 2004), apresentado a seguir, que requer o equacionamento das trocas de calor entre os diversos segmentos do manequim e o ambiente, a calibração do manequim e a utilização de diagrama de sensação térmica.

\section{Equacionamento das trocas de calor}

Para manequins sem sudorese, a determinação de temperaturas equivalentes, $T_{\text {eq, é baseada na }}$ transferência de calor por convecção e radiação em cada segmento, dada pelas Equações 4 e 5:

$$
\begin{aligned}
& R=h_{r}\left(T_{s}-\bar{T}_{r}\right) \\
& C=h_{c}\left(T_{s}-T_{a r}\right)
\end{aligned}
$$

Onde:

$\mathrm{C}=$ troca de calor por condução $\left[\mathrm{W} / \mathrm{m}^{2}\right]$;

$\mathrm{R}=$ troca de calor por radiação $\left[\mathrm{W} / \mathrm{m}^{2}\right]$;

$\mathrm{h}_{\mathrm{r}}=$ coeficiente de transferência de calor por radiação $\left[\mathrm{W} / \mathrm{m}^{2} \mathrm{~K}\right]$;

$\mathrm{h}_{\mathrm{c}}=$ coeficiente de transferência de calor por convecção $\left[\mathrm{W} / \mathrm{m}^{2} \mathrm{~K}\right]$;

$\mathrm{T}_{\mathrm{s}}=$ temperatura da superfície $\left[{ }^{\circ} \mathrm{C}\right]$;
$\bar{T}_{r}=$ temperatura radiante média [ $\left.{ }^{\circ} \mathrm{C}\right]$; e

$\mathrm{T}_{\mathrm{ar}}=$ temperatura do ar ambiente $\left[{ }^{\circ} \mathrm{C}\right]$.

Uma vez que as trocas de calor por convecção e radiação ocorrem simultaneamente, a temperatura equivalente, $\mathrm{T}_{\text {eq, }}$ é função dessas trocas de calor e é dada por:

$T_{e q}=T_{s}-\frac{\dot{Q}}{h}$

Eq. 6

Onde:

$\dot{Q}=\mathrm{R}+\mathrm{C}$ : troca de calor por radiação e convecção $\left[\mathrm{W} / \mathrm{m}^{2}\right]$;

$\mathrm{T}_{\text {eq }}=$ temperatura equivalente $\left[{ }^{\circ} \mathrm{C}\right]$;

$\mathrm{T}_{\mathrm{s}}=$ temperatura da superfície $\left[{ }^{\circ} \mathrm{C}\right]$; e

$\mathrm{h}=$ coeficiente de transferência de calor combinado, convecção e radiação [W/m² K].

\section{Procedimento de calibração do manequim}

Para aplicar-se o conceito de temperatura equivalente (Figura 4) o manequim precisa ser calibrado em um ambiente térmico padrão com as mesmas vestimentas e na mesma posição que será usado para avaliar o ambiente térmico em estudo. As vestimentas afetam o coeficiente de transferência de calor sensível e, portanto, devem ser escolhidas adequadamente para a situação a ser avaliada e devem ser mantidas durante todo o processo de avaliação.

O procedimento de calibração consiste na determinação de coeficientes de transferência de calor combinado, h, da Equação 6, para os diversos segmentos do corpo em um ambiente padrão. Para satisfazer o conceito de temperatura equivalente, o ambiente padrão é um ambiente homogêneo com temperatura de bulbo seco igual à temperatura 
radiante média, $\mathrm{T}_{\mathrm{ar}}=\bar{T}_{r}$, e com velocidade do ar próximo de zero $(\mathrm{V}<0,1 \mathrm{~m} / \mathrm{s})$. Nessas condições tem-se $\mathrm{T}_{\mathrm{ar}}=\bar{T}_{r}=\mathrm{T}_{\mathrm{eq}}$ (Figura 4).

Uma vez satisfeitas essas condições em ensaio realizado em câmara climatizada e obtidas condições de regime permanente nas trocas de calor do manequim com o ambiente, são realizadas leituras de temperaturas superficiais, $\mathrm{T}_{\mathrm{s}}$, e de fluxo de calor, $\dot{Q}$, para cada segmento do corpo e calculados valores de coeficientes de troca de calor, hcal, que são os coeficientes de troca de calor da calibração, dados pela Equação 7:

$h_{c a l}=\frac{\dot{Q}}{T_{s}-T_{e q}}$

Os valores de coeficientes de transferência de calor da calibração, hcal, serão os valores de coeficiente de troca de calor, h, da Equação 6 no cálculo das temperaturas equivalentes no ambiente real.

Uma vez calibrado o manequim para a posição e vestimenta do ensaio real, o método de avaliação consiste em posicionar o manequim no ambiente real e medir os fluxos de calor e as temperaturas superficiais de cada segmento nesse ambiente. Por meio da Equação 8 é possível determinar a $\mathrm{T}_{\mathrm{eq}}$ para cada segmento ou para o corpo todo utilizando os valores de hcal da calibração e os novos valores de $\dot{Q}$ e $\mathrm{T}_{\mathrm{s}}$.

$T_{e q}=T_{s}-\frac{\dot{Q}}{h_{c a l}}$

Eq. 8
As temperaturas equivalentes assim determinadas são indicadores do nível de afastamento entre as condições do ambiente e as condições correspondentes a uma sensação térmica de neutralidade.

\section{Diagramas de sensação térmica}

Para avaliar-se quão distantes as condições do ambiente encontram-se de condições correspondentes a uma sensação térmica de neutralidade é necessário plotar os resultados das temperaturas equivalentes, $\mathrm{T}_{\text {eq }}$, em diagramas de sensação térmica (Figura 5).

Os valores de $\mathrm{Y}$ da Figura 5 correspondem às temperaturas equivalentes, $\mathrm{T}_{\mathrm{eq}}$, e os valores de $\mathrm{X}$ aos segmentos do corpo (manequim). O segmento 17 corresponde à $\mathrm{T}_{\text {eq }}$ para o corpo como um todo. Os números 1 a 5 representam as faixas de sensação térmica, onde 1 representa sensação de frio, 2 levemente frio, 3 confortável, 4 levemente quente e 5 quente.

\section{Método}

No estudo do desconforto térmico local foram realizados ensaios em três salas cirúrgicas com três diferentes sistemas de ventilação e quatro condições de ensaio, conforme apresentado no Quadro 1, juntamente com o tipo de cirurgia realizada.

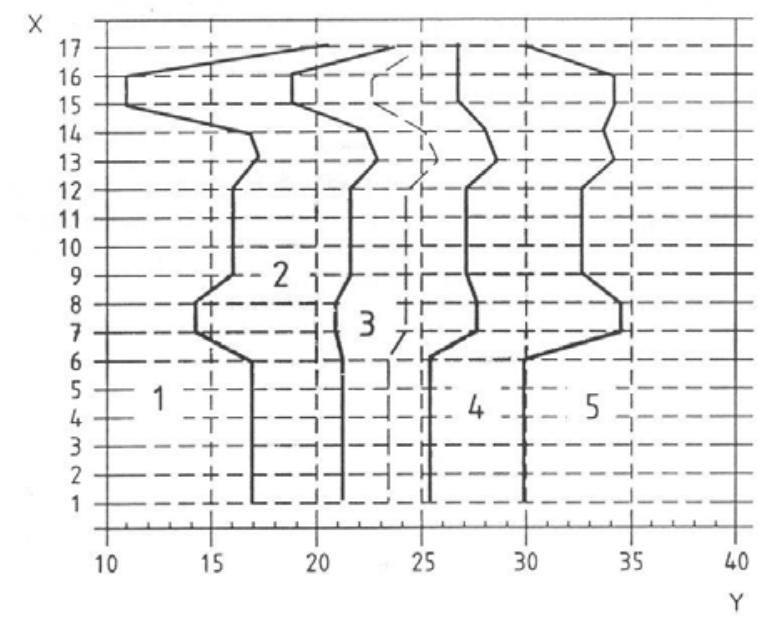

Figura 5 - Diagrama de sensação térmica

Fonte: ISO 14505-2 (INTERNATIONAL..., 2004). 


\begin{tabular}{|l|l|l|}
\hline \multicolumn{1}{|c|}{ Condições } & \multicolumn{1}{|c|}{$\begin{array}{c}\text { Características do } \\
\text { sistema de ventilação }\end{array}$} & Tipo de cirurgia \\
\hline Sala Cirúrgica 1/Ensaio A & Sistema desligado & Hérnia e varizes \\
\hline Sala Cirúrgica 1/Ensaio B & $\begin{array}{l}\text { Sistema com } \\
\text { insuflamento pela parede } \\
\text { na parte superior e } \\
\text { retorno pela mesma } \\
\text { parede na parte inferior } \\
\text { no lado oposto. }\end{array}$ & Hérnia e varizes \\
\hline Sala Cirúrgica 2/Ensaio C & $\begin{array}{l}\text { Sistema com } \\
\text { insuflamento pelo teto e } \\
\text { retorno pelo teto. }\end{array}$ & Cesarianas \\
\hline Sala Cirúrgica 3/Ensaio D & $\begin{array}{l}\text { Sistema com fluxo } \\
\text { unidirecional (“laminar”) }\end{array}$ & $\begin{array}{l}\text { Ortopédicas de } \\
\text { pequeno porte }\end{array}$ \\
\hline
\end{tabular}

\section{Quadro 1 - Condições de ensaio}

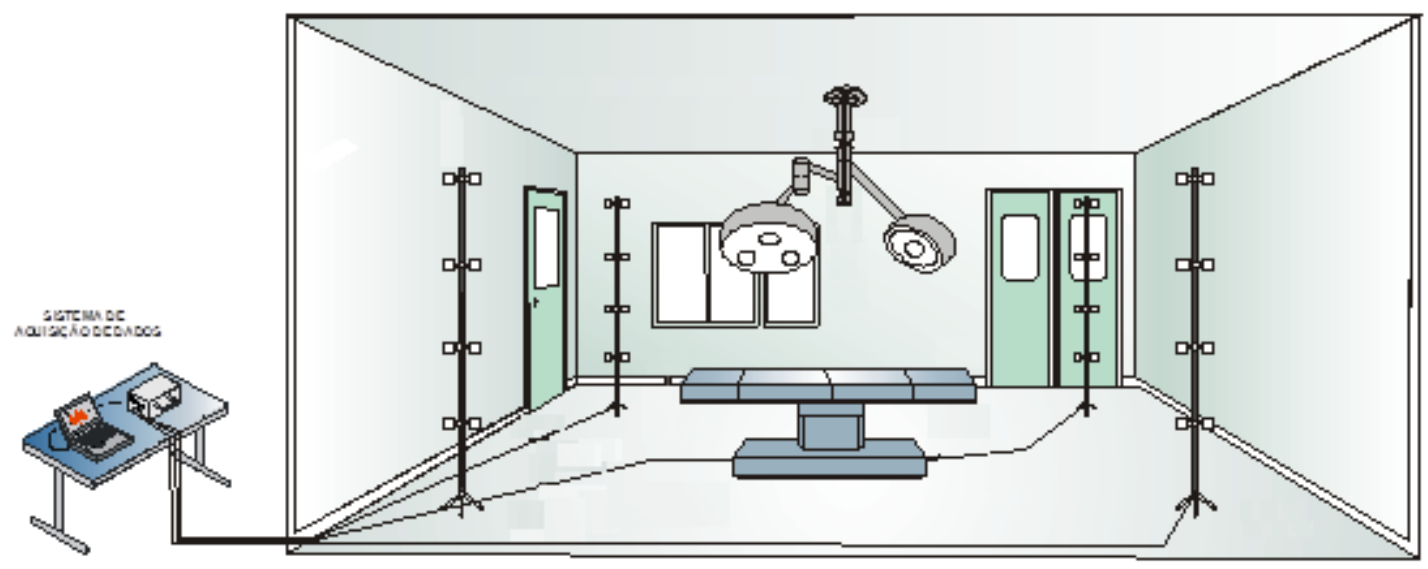

Figura 6 - Exemplo de disposição dos equipamentos nas salas cirúrgicas

O estudo de condições de desconforto com o sistema de ventilação desligado foi possível de ser realizado em procedimentos cirúrgicos bastante simples e com risco de infecção extremamente baixo (FELIX, 2008; FELIX et al., 2010). Além disso, para diminuir o efeito da interferência e o risco da presença de equipamentos durante o procedimento cirúrgico, todos os ensaios ocorreram durante a realização de cirurgias simples com risco de infecção baixo, conforme pode ser verificado no Quadro 1.

Para cada uma das quatro condições de ensaio foram realizadas medições e avaliações subjetivas em aproximadamente oito cirurgias, com equipes cirúrgicas compostas de quatro membros, cirurgião, instrumentador, anestesista e enfermeiro, com larga experiência na realização de procedimentos cirúrgicos em suas respectivas funções.

Um questionário baseado no trabalho de Mora, English e Athienitis (2001) foi aplicado ao término de cada cirurgia. Nesse questionário os membros da equipe cirúrgica responderam a questões referentes ao desconforto devido a correntes de ar e à proximidade de superfícies frias (anestesistas e enfermeiros) e de desconforto devido a assimetrias da temperatura radiante causadas pelo foco cirúrgico (cirurgiões e instrumentadores). Ao todo foram respondidos 120 questionários relativos às respostas dos quatro membros das equipes cirúrgicas em mais de 30 cirurgias realizadas (aprox. oito cirurgias em cada uma das quatro condições de ensaio do Quadro 1).

Durante as cirurgias foram medidas temperaturas e velocidades do ar em quatro alturas - 0,1 m; 0,6 m; 1,1 m; e 1,7 m do nível do piso -, previstas na norma ASHRAE 55 (ABNT, 2004), e em quatro pontos diferentes ao longo das salas cirúrgicas. A Figura 6 apresenta uma ilustração da disposição dos equipamentos nas salas cirúrgicas.

Os valores de temperaturas do ar foram utilizados para verificação da diferença vertical de temperatura entre os pés e a cabeça (estratificação da temperatura) durante as cirurgias. Os valores de velocidade do ar e de temperatura do ar a 1,70 m (altura da cabeça e tronco de pessoa em pé) foram 
utilizados para análise do percentual de insatisfação devido a correntes de ar, DR Draught Rate, utilizando a Equação 2.

A medição das variáveis ambientais foi realizada segundo procedimentos e métodos de medição apresentados na norma ISO 7726 (INTERNATIONAL..., 1998), e os sensores utilizados para as medições de temperatura foram termômetros de resistência; para as medições de velocidades, foram utilizados anemômetros omnidirecionais. Os equipamentos de medição foram calibrados e atendem aos requisitos preconizados na norma ISO 7726 (INTERNATIONAL..., 1998), conforme apresentado na Tabela 1.

Os ensaios foram realizados em salas cirúrgicas com umidades relativas do ar variando entre $45 \%$ e $50 \%$, que são valores típicos em ambientes dotados de sistema de ar condicionado central. Durante os ensaios também foram medidas temperaturas de globo para cálculo de temperaturas radiantes médias, cujos valores foram utilizados para a determinação de condições de conforto térmico apresentadas em Felix et al. (2010).

Depois de realizadas as medições de variáveis ambientais e aplicados os questionários, foram realizados levantamentos de condições de desconforto térmico local com o uso do manequim com sensores aquecidos, simulando o cirurgião, que é o profissional mais exigido da equipe cirúrgica.

Devido à impossibilidade de manter o manequim ocupando o mesmo espaço do cirurgião ou mesmo próximo deste sem causar problemas durante o procedimento cirúrgico, os ensaios com o manequim foram realizados depois, em separado. É importante ressaltar, contudo, que esse procedimento não compromete a análise dos resultados, pois permite que o manequim seja posicionado exatamente no mesmo local ocupado anteriormente pelo cirurgião, sob influência direta dos focos cirúrgicos.

Os ensaios com o manequim foram realizados na sala cirúrgica com sistema de insuflamento pela parede na parte superior e retorno pela mesma parede na parte inferior no lado oposto (Figura 7, Ensaio B do Quadro 1), com temperatura do ar no ambiente em torno de $22{ }^{\circ} \mathrm{C}$, considerando potências dissipadas de $0 \mathrm{~W}$ (foco cirúrgico desligado), $200 \mathrm{~W}, 300 \mathrm{~W}, 400 \mathrm{~W}$ e $500 \mathrm{~W}$.

No procedimento de calibração e de ensaio com o uso do manequim todos os segmentos do corpo foram controlados para dissipar a mesma quantidade de calor, $\dot{Q}$, de $90 \mathrm{~W} / \mathrm{m}^{2}$, representando a atividade realizada pelo cirurgião (1,6 met). $\mathrm{O}$ valor de $90 \mathrm{~W} / \mathrm{m}^{2}$ (1,6 met) foi arbitrado e corresponde ao valor de metabolismo de cirurgiões utilizado por Mora, English e Athienitis (2001).

\begin{tabular}{l|l|l}
\hline \multicolumn{1}{c|}{ Variáveis } & \multicolumn{1}{c|}{ Faixa de medição } & \multicolumn{1}{c}{ Precisão } \\
\hline Temperatura do ar & 0 a 50 0C & $\pm 0,3{ }^{\circ} \mathrm{C}$ \\
Velocidade do ar & 0 a $1 \mathrm{~m} / \mathrm{s}$ & $\pm\left(0,04+3 \% \mathrm{~V}_{\mathrm{ar}}\right) \mathrm{m} / \mathrm{s}$ \\
\hline
\end{tabular}

Tabela 1 - Características dos instrumentos de medição utilizados

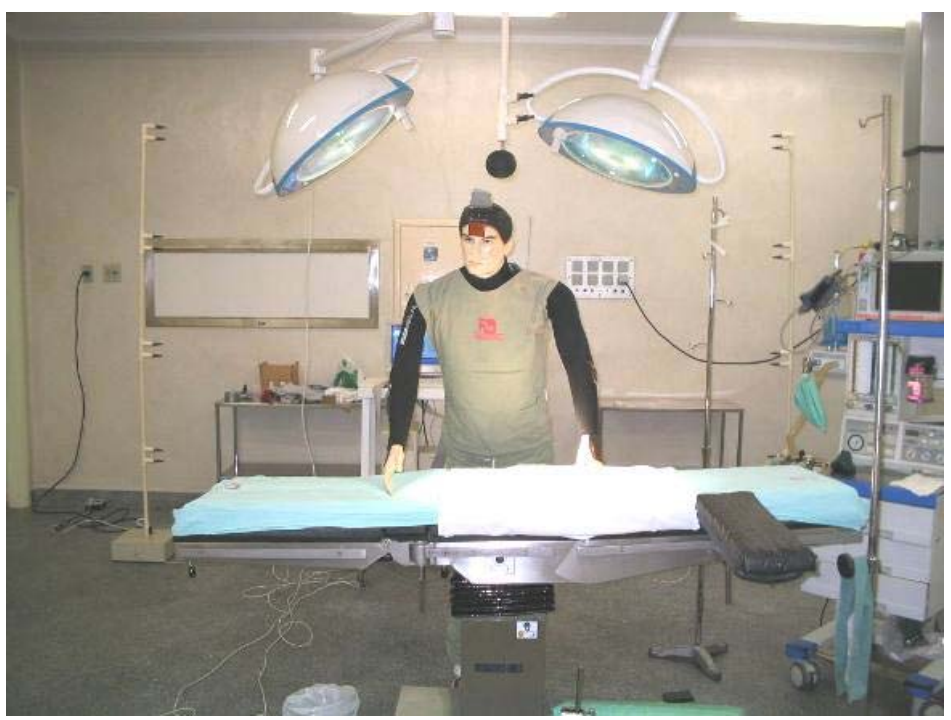

Figura 7 - Manequim posicionado na sala cirúrgica do Ensaio B, Tabela 1, simulando o cirurgião 
No ambiente padrão uniforme representado na Figura 4, com temperatura equivalente, $\mathrm{T}_{\mathrm{eq}}=24$ ${ }^{\circ} \mathrm{C}$, foram medidos valores de temperaturas superficiais, $\mathrm{T}_{\mathrm{s}}$, e determinados os valores de coeficiente de troca de calor de calibração, hcal, utilizando-se a equação 7.

Uma vez calibrado o manequim para a posição e vestimenta do ensaio com determinação dos valores hcal, foram medidas novas temperaturas da superfície, $T_{s}$, em cada segmento do manequim na sala cirúrgica (Figura 7) para a mesma troca de calor $\dot{Q}=90 \mathrm{~W} / \mathrm{m}^{2}$ em cada segmento. Com esses valores foram determinadas as temperaturas equivalentes, $\mathrm{T}_{\text {eq }}$, em cada segmento utilizando a Equação 8. Uma vez determinados os valores de $\mathrm{T}_{\text {eq }}$, eles foram plotados no gráfico da Figura 5, cujos resultados são apresentados e discutidos no próximo item.

\section{Aanálise de resultados}

Na Figura 8 são apresentados resultados de medição de diferença vertical de temperatura entre os pés e a cabeça próximo à equipe cirúrgica em cada tipo de sala cirúrgica/ensaio do Quadro 1. Os valores apresentados referem-se à média dos valores médios ao longo das cirurgias em cada uma das quatro condições de ensaio estudadas.

Uma vez que para cada condição de ensaio foram feitas várias medições e em vários pontos ao longo da sala, o gráfico da Figura 8 apresenta os perfis de temperatura nos locais onde foram encontradas as maiores diferenças verticais de temperatura do ar para cada ensaio.

A análise dos resultados de perfis de temperatura apresentados na Figura 8 mostra que não ocorreram grandes diferenças verticais de temperatura nas salas cirúrgicas, com valores bastante inferiores ao valor limite de $3{ }^{\circ} \mathrm{C}$ preconizado nas normas ASHRAE 55 (AMERICAN..., 2004), ISO 7730 (INTERNATIONAL..., 2005) e NBR 16401 (ABNT, 2008).

Na Tabela 2 são apresentados resultados de medição de temperaturas e velocidades do ar e do cálculo de intensidade de turbulência e de percentual de insatisfação (DR) devido a correntes de ar a uma altura de $1,70 \mathrm{~m}$ do piso (região da cabeça e do tórax de pessoas em pé). Os valores de temperatura e velocidade do ar apresentados referem-se à média dos valores médios ao longo das cirurgias em cada condição de ensaio estudada próximo à posição onde ficam os anestesistas e enfermeiros. A escolha da posição ocupada por anestesistas e enfermeiros para o cálculo do DR deve-se ao fato de estes apresentarem maior risco de desconforto por correntes de ar devido, principalmente a seu menor nível de atividade durante os procedimentos cirúrgicos.

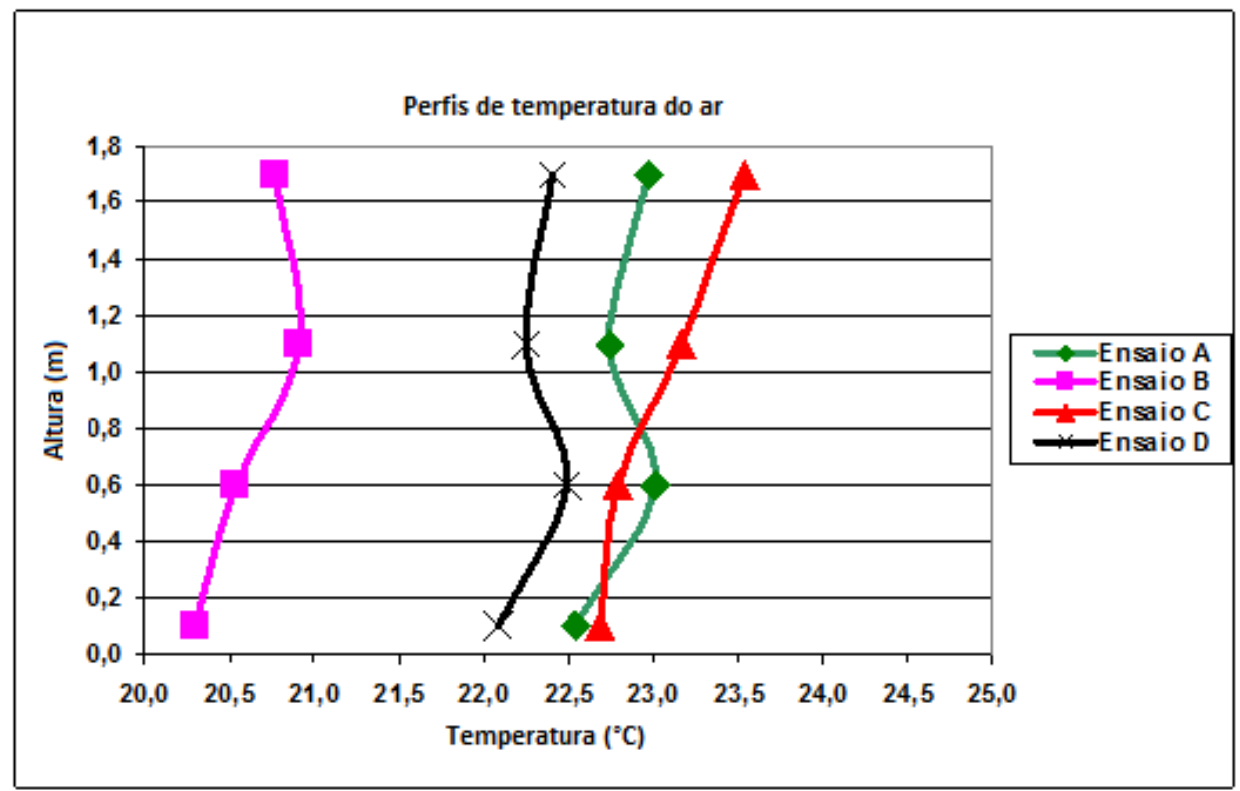

Figura 8 - Perfis de temperatura do ar 
Tabela 2 - Resultados de medições de temperaturas e velocidades do ar e de cálculo de intensidade de turbulência e de percentual de insatisfação devido a correntes de ar (DR - draught rate) na região onde ficam os anestesistas e os enfermeiros

\begin{tabular}{l|c|c|c|c}
\hline Condições & $\begin{array}{c}\text { Temperatura } \\
\left({ }^{\circ} \mathbf{C}\right)\end{array}$ & $\begin{array}{c}\text { Velocidade } \\
(\mathbf{m} / \mathbf{s})\end{array}$ & $\begin{array}{c}\text { Intensidade de } \\
\text { turbulência (\%) }\end{array}$ & $\begin{array}{c}\text { DR } \\
(\mathbf{\%})\end{array}$ \\
\hline Ensaio A & 23,5 & 0,10 & 50 & 8,2 \\
Ensaio B & 20,3 & 0,25 & 28 & 29,0 \\
Ensaio C & 22,9 & 0,30 & 27 & 28,7 \\
Ensaio D & 22,2 & 0,20 & 25 & 18,0 \\
\hline
\end{tabular}

Tabela 3 - Respostas referentes ao desconforto devido à proximidade de superfícies frias e por correntes de ar

\begin{tabular}{l|cccc|cccc}
\hline \multirow{2}{*}{ Causas } & \multicolumn{5}{c|}{ Anestesistas } & \multicolumn{4}{c}{ Enfermeiros } \\
\cline { 2 - 8 } & $\begin{array}{c}\text { Ensaio } \\
\text { A }\end{array}$ & $\begin{array}{c}\text { Ensaio } \\
\text { B }\end{array}$ & $\begin{array}{c}\text { Ensaio } \\
\text { C }\end{array}$ & $\begin{array}{c}\text { Ensaio } \\
\text { D }\end{array}$ & $\begin{array}{c}\text { Ensaio } \\
\text { A }\end{array}$ & $\begin{array}{c}\text { Ensaio } \\
\text { B }\end{array}$ & $\begin{array}{c}\text { Ensaio } \\
\text { C }\end{array}$ & $\begin{array}{c}\text { Ensaio } \\
\text { D }\end{array}$ \\
\hline $\begin{array}{l}\text { Desconforto } \\
\text { causado por } \\
\text { correntes de ar }\end{array}$ & $10 \%$ & $35 \%$ & $35 \%$ & $0 \%$ & $0 \%$ & $20 \%$ & $20 \%$ & $30 \%$ \\
\hline $\begin{array}{l}\text { Desconforto } \\
\text { devido à } \\
\text { proximidade de } \\
\text { superfícies frias }\end{array}$ & $25 \%$ & $20 \%$ & $20 \%$ & $0 \%$ & $25 \%$ & $35 \%$ & $20 \%$ & $0 \%$ \\
\hline
\end{tabular}

Tabela 4 - Respostas referentes ao desconforto devido ao calor do foco cirúrgico

\begin{tabular}{l|c|c|c|c|c|c|c|c}
\hline \multirow{2}{*}{ Causa } & \multicolumn{4}{c|}{ Cirurgiões } & \multicolumn{4}{c}{ Instrumentadores } \\
\cline { 2 - 8 } & $\begin{array}{c}\text { Ensaio } \\
\text { A }\end{array}$ & $\begin{array}{c}\text { Ensaio } \\
\text { B }\end{array}$ & $\begin{array}{c}\text { Ensaio } \\
\text { C }\end{array}$ & $\begin{array}{c}\text { Ensaio } \\
\text { D }\end{array}$ & $\begin{array}{c}\text { Ensaio } \\
\text { A }\end{array}$ & $\begin{array}{c}\text { Ensaio } \\
\text { B }\end{array}$ & $\begin{array}{c}\text { Ensaio } \\
\text { C }\end{array}$ & $\begin{array}{c}\text { Ensaio } \\
\text { D }\end{array}$ \\
\hline $\begin{array}{l}\text { Desconforto } \\
\text { devido ao } \\
\text { foco } \\
\text { cirúrgico }\end{array}$ & $20 \%$ & $70 \%$ & $60 \%$ & $85 \%$ & $0 \%$ & $0 \%$ & $35 \%$ & $25 \%$ \\
\hline
\end{tabular}

Os resultados apresentados na Tabela 2 mostram que todos os valores de percentual de insatisfação (DR) são superiores a 15\%, que é o percentual limite utilizado nas normas ASHRAE 55 (ABNT, 2004), ISO 7730 (INTERNATIONAL..., 2005) e NBR 16401 (ABNT, 2008) na definição das velocidades máximas do ar em ambientes climatizados, exceto no Ensaio A com o sistema de climatização desligado.

Nas Tabelas 3 e 4 são apresentados os resultados obtidos a partir da aplicação de questionário abordando questões referentes ao desconforto de anestesistas e enfermeiros a correntes de ar e devido a superfícies frias (Tabela 3) e ao desconforto de cirurgiões e instrumentadores devido ao calor dos focos cirúrgicos (Tabela 4).

Os resultados de desconforto causado por correntes de ar obtidos a partir dos questionários apresentados na Tabela 3 mostram resultados similares àqueles obtidos a partir de medições apresentados na Tabela 2. Adicionalmente, os resultados obtidos a partir de questionários permitem captar diferenças entre anestesistas e enfermeiros. Essas diferenças estão relacionadas com as atividades que cada um exerce durante $\mathbf{o}$ procedimento cirúrgico e a posição em que cada um se encontra no ambiente em relação ao insuflamento de ar. Normalmente, os enfermeiros têm maior liberdade de movimentação, enquanto os anestesistas ficam mais parados, monitorando os sinais vitais do paciente, o que os deixa mais sujeitos ao risco de correntes de ar ou, excepcionalmente, fora desse risco, como ocorre no caso do ensaio $\mathrm{D}$, onde os anestesistas permanecem fora da região de insuflamento de ar do sistema de fluxo unidirecional.

Os resultados de desconforto devido ao foco cirúrgico (Tabela 4) mostram grande desconforto por parte dos cirurgiões, com percentuais de insatisfação significativamente maiores que o dos instrumentadores. Isso ocorre porque os cirurgiões apresentam maior nível de atividade e têm de desenvolver essas atividades diretamente sob o efeito dos focos cirúrgicos na iluminação da ferida 
cirúrgica, cuja potência era de $600 \mathrm{~W}$ em todos os ensaios.

Finalmente, na Figura 9 são apresentados os resultados de temperaturas equivalentes obtidos com o uso do manequim para os diversos segmentos do corpo considerando diferentes potências do foco cirúrgico. Na Figura 10 é destacado o aumento significativo da temperatura equivalente para a cabeça em função da potência do foco cirúrgico.

Analisando-se os resultados apresentados no diagrama de sensação térmica da Figura 9 é possível verificar a influência significativa da potência dissipada pelo foco cirúrgico no desconforto local dos cirurgiões, corroborando os resultados da análise subjetiva apresentados na Tabela 4.

Verifica-se ainda da análise da Figura 9 que o aumento da potência do foco cirúrgico também tem influência nas condições de desconforto térmico local nos demais segmentos do corpo. Embora neste caso em particular ocorra uma melhora nas condições de conforto nos diversos segmentos do corpo com menor desconforto devido ao frio, o desconforto causado pelo foco cirúrgico é significativamente maior.

\section{Conclusões}

Inicialmente, é importante lembrar que os resultados de desconforto térmico local em salas cirúrgicas aqui apresentados referem-se a situações em que foram realizados procedimentos cirúrgicos simples, que demandam menor nível de estresse e de atividades da equipe cirúrgica. Situações mais complexas e procedimentos cirúrgicos mais demorados podem significar um nível de desconforto ainda maior do que o dos resultados obtidos no presente trabalho.

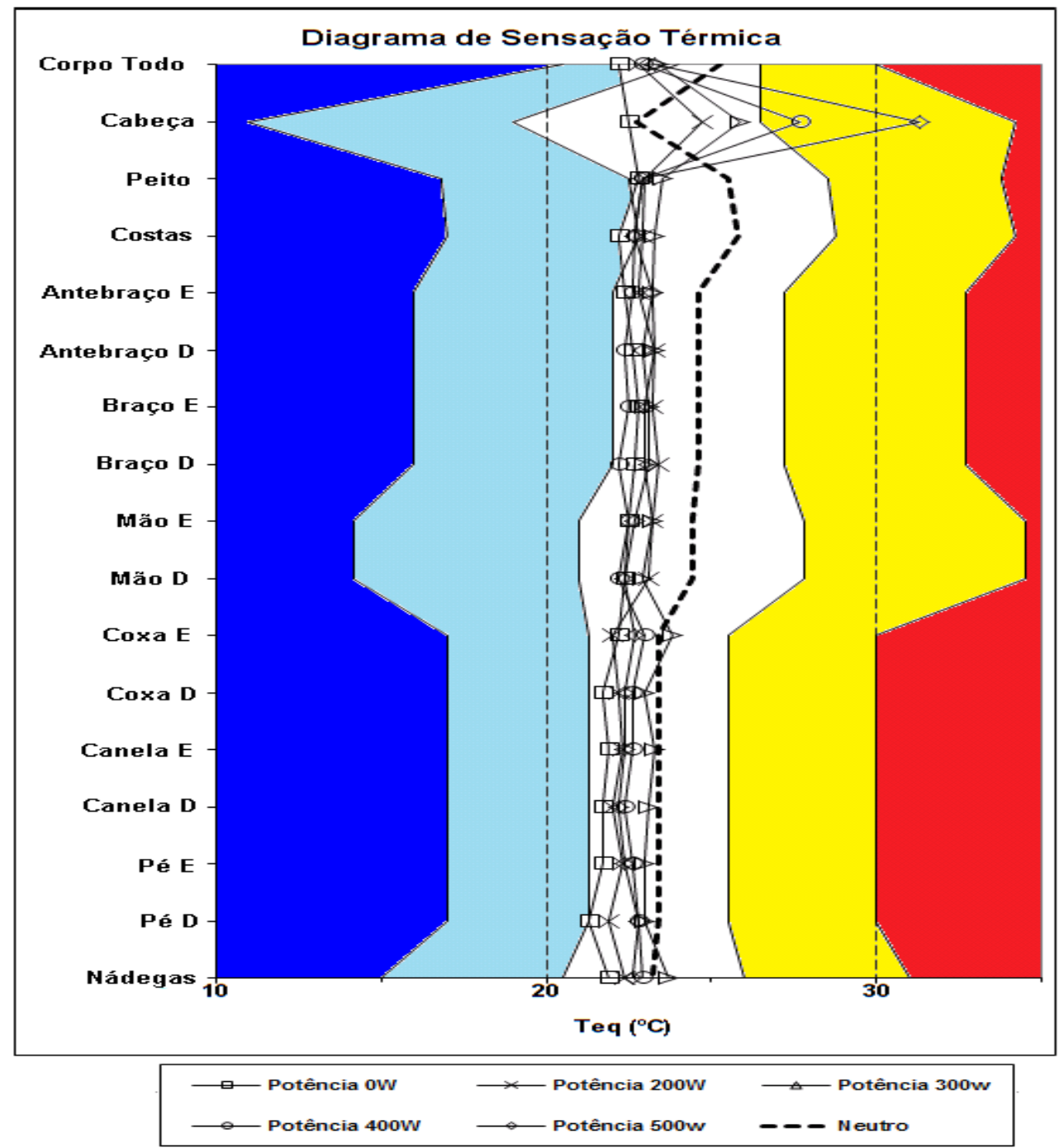

Figura 9 - Diagrama de sensação térmica do cirurgião para diferentes potências do foco cirúrgico 
Temperatura Equivalente da Cabeça

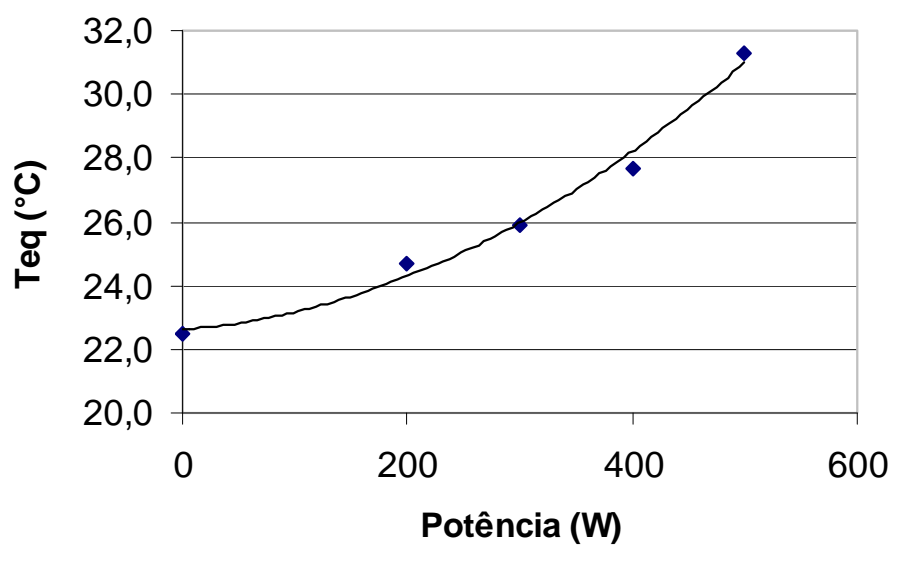

Figura 10 - Temperatura equivalente da cabeça em função da potência do foco cirúrgico

Os resultados obtidos mostram que na região onde se encontram os cirurgiões e os instrumentadores existe uma grande tendência ao desconforto local devido à radiação emitida pelos focos cirúrgicos e que o cirurgião é o profissional mais afetado. Os enfermeiros e os anestesistas estão mais sujeitos ao desconforto devido a correntes de ar e à proximidade de superfícies frias.

Resultados da avaliação subjetiva mostraram níveis de insatisfação de até 35\% dos anestesistas e enfermeiros com correntes de ar e de até $85 \%$ dos cirurgiões com o calor do foco cirúrgico. Resultados similares foram obtidos a partir da medição de variáveis ambientais e com o uso de manequim.

Por tratar-se do profissional mais importante da equipe cirúrgica, com maior grau de responsabilidade, é de fundamental importância prover melhores condições de conforto para o cirurgião. Para tal, a solução seria a utilização de focos cirúrgicos que dissipassem menor quantidade de calor, conforme mostrado nas Figuras 9 e 10. Na impossibilidade de fazer-se isso, o ar deveria ser insuflado com temperaturas menores e/ou vazões maiores na região onde ficam os cirurgiões. Vazões maiores podem propiciar melhor resultado, porque não haveria necessidade de temperaturas muito baixas do ar, diminuindo o risco de desconforto local devido a correntes de ar.

Embora o bem-estar da equipe cirúrgica seja muito importante para o sucesso do procedimento cirúrgico, é importante ressaltar que os riscos de contaminação aérea não podem ser negligenciados. Assim, a solução do problema deve considerar ainda, simultaneamente, os efeitos da movimentação do ar na diminuição da possibilidade de contaminação aérea e na obtenção das melhores condições possíveis de conforto térmico.
Outro aspecto importante a destacar é o uso de manequins para avaliação de condições de conforto e desconforto térmico local em situações com variações significativas no ambiente, como é o caso das salas cirúrgicas. A apresentação dos resultados em diagrama de sensação térmica, a exemplo daqueles da Figura 9, permite que se possa fazer a um só tempo a análise de condições de conforto térmico para o corpo como um todo e em cada um dos segmentos do corpo. Esse procedimento pode significar economia de tempo e uma análise mais precisa e detalhada das condições de conforto nesse tipo de ambiente.

Por fim, o uso de diferentes ferramentas de análise de desconforto térmico local pode auxiliar na busca de proverem-se condições de conforto térmico as melhores possíveis para todos os membros da equipe cirúrgica.

\section{Referências}

\section{AMERICAN INDUSTRIAL HYGIENE ASSOCIATION. Field Guide for the Determination of Biological Contaminants in Environmental Samples. Virginia: American Industrial Hygiene Association, 1996.}

\section{AMERICAM SOCIETY OF HEATIN, REFRIGERATING AND AIR-} CONDITIONINGENGINEERS. Standard 55: thermal environmental conditions for human occupancy. Atlanta, 2004.

\section{AMERICAM SOCIETY OF HEATIN, REFRIGERATING AND AIR- \\ CONDITIONINGENGINEERS. Handbook of}

Fundamentals. Atlanta: American Society of Heating, Refrigerating and Air Conditioning Engineers, Inc., 2009. 
ASSOCIAÇÃO BRASILEIRA DE NORMAS TÉCNICAS. NBR 16401-2: instalações de ar condicionado: sistemas centrais e unitários: parte 2: parâmetros de conforto térmico. Rio de Janeiro, 2008.

BEGGS, C. B. The Airborne Transmission of Infection in Hospital Buildings: fact or fiction? Indoor and Built Environment, v. 12, p. 9-18, 2003.

CASSEY, J. et al. Influence of Control Variables on Mannequin Temperature in a Pediatric

Operating Theatre. Pediatric Anesthesia, v. 14, p. 130-134, 2004.

FANGER, P. O. Thermal Comfort: analysis and applications in environmental engineering. New York: McGraw-Hill, 1972.

FANGER, P. O. et al. Air Turbulence and Sensation of Draught. Energy and Buildings, v 12, p. 21-39, 1988.

FELIX, V. B. Condições de Conforto Térmico e de Desconforto Local em Salas Cirúrgicas. $80 \mathrm{f}$. São Paulo, 2008. Dissertação (Mestrado em Engenharia Mecânica) - Escola Politécnica, Universidade de São Paulo, São Paulo, 2008.

FELIX, V. B. et al. The Effect of Surgical Lights Power on Staff Thermal Discomfort Conditions. In: International Conference on Biomedical Engineering, 12., Singapore, 2005. Proceedings... Singapore, 2005.

FELIX, V. B. et al. Avaliação de Conforto Térmico em Ambientes Cirúrgicos Utilizando Método de Fanger e Temperaturas Equivalentes. Ambiente Construído, Porto Alegre, v. 10 n. 4, p. 79-68, out./dez. 2010.

GAMEIRO DA SILVA, M. C. Measurements of Comfort in Vehicles. Measurement Science and Technology, v. 13, n. 6, p. 41-60, 2002.

HOWORTH, F. Prevention of Airborne Infection During Surgery. ASHRAE Transactions, v. 99, n. 1, p. 725-734, 1993.

INTERNATIONAL ORGANIZATION FOR STANDARDIZATION. ISO 7726: thermal environments: instruments and methods for measuring physical quantities. Geneva, 1998.

INTERNATIONAL ORGANIZATION FOR STANDARDIZATION. ISO 7730: ergonomics of the thermal environment: analytical determination and interpretation of thermal comfort using calculation of the PMV and PPD indices at local thermal criteria. Geneva, 2005.
INTERNATIONAL ORGANIZATION FOR STANDARDIZATION. ISO 14505-2:

ergonomics of the thermal environment: evaluation of thermal environment in vehicle: part2: determination of equivalent temperature. Geneva, 2004.

MADSEN, T. L.; OLESEN, B. W.; KRISTENSEN, N. K. Comparison Between Operative and Equivalent Temperature and Typical Indoor Conditions. ASHRAE Transactions, v. 90, n. 1, p. 1077-1090, 1984. MORA, R.; ENGLISH, M. J. M.; ATHIENITIS, A. K. Assessment of thermal comfort during surgical operations. ASHRAE Transactions, v. 107, n. 1, p. 52-62, 2001.

NILSSON, H. O. Comfort Climate Evaluation With Thermal Manikin Methods and Computer Simulation Modes. 202 f. Stockholm, 2004. PhD Thesis - Department of Technology and Built Environment, University of Gaule, Stockholm, 2004.

PEREIRA, M. L.; TRIBESS, A. A Review of Air Distribution Patterns in Surgery Rooms Under Infection Control Focus. Engenharia Térmica, v. 4, n. 2, p. 113-121, 2005.

WHYTE, W. et al. The Relative Importance of Routes and Sources of Wound Contamination During General Surgery II Airborne. Journal of Hospital Infection, v. 22, n. 2, p. 41-54, 1992.

WHYTE, W.; HODGSON, R.; TINKLER, J. The Importance of Airborne Bacterial Contamination of Wounds. Journal of Hospital Infection, v. 3, n. 2, p. 123-135, 1982.

WYON, D. P.; LIDWELL, O. M.; WILLIANS, R. E. O. Thermal Comfort During Surgical Operations. Journal of Hygiene, v. 66, p. 229248, 1968.

\section{Agradecimentos}

Os autores Victor Barbosa Felix, Marcelo Luiz Pereira e Arlindo Tribess agradecem ao CNPq pela concessão de bolsa de mestrado, doutorado e de pesquisa respectivamente. Danilo de Moura agradece à FAPESP pela concessão de bolsa de mestrado. 